except when they cross the resonance plane where their local cyclotron Prequency is equal to the i'requency of the applied microwaves. At each rescrance crossing, the perpendicular velocity is abruptly changed by an amount proportional to the applied rms electric field, inversely proportional to the square root of the parallel velocity, and proportional to the cosine of a randomly chosen phase angle ${ }^{3}$ :

$$
\Delta \mathrm{v}_{\perp} \propto \sqrt{\overline{\mathbb{E}_{\perp}^{2}} / \mathrm{v}_{11}} \cos \phi \text {. }
$$

This method of simulating the electron cyclotron heating has been verified in the nonrelativistic limit in other numerical calcuiations. 4 The applied electric field is continually readjusted so as to maintair total absorption of a given input microwave power.

The space charge electric field is calculated from Poisson's equation using a least square fit to the lowest order spatial Fourier component:

$$
E_{11}(2)=E_{0} \sin \pi R / L \text {. }
$$

AII quantities are assumed uniform in the airection perpendicular to the magnetic field. The ions are accelerated only by the space charge electric field parillel to $\vec{B}$. The ions never acquire any perpendicular energy. The external rf electric field does not enter explicitly in the equations of motion for either the electrons or the ions.

\section{Typical Results}

A wide variety of cases have been examined, but in the interest of brevity, only one typical case will be discussed. The parameters are somewhat unrealistic $a$; is often the case with a finite computing budget and a diversity of time scales:

microwave frequency: $\omega / 2 \pi=10 \mathrm{GHz}$

microwave power density: $\frac{\mathrm{dP}}{\mathrm{dV}}=1.23 \mathrm{~kW} / \mathrm{cm}^{3}$

microwave pulse Iength: $T=0.048 \mu \mathrm{sec}$

plasma density: $\mathbf{n}=1.23 \times 10^{9} \mathrm{csi}^{-3}$

initial electron temperature: $\mathrm{kT}_{\mathrm{e}}=1 \mathrm{keV}$

mirror ratio: $R=2$

mirror length: $2 \pi I=60 \mathrm{~cm}$

resonance plane: $\pi I_{0}=15 \mathrm{~cm}$

magnetic field at resonance: $B_{0}=3.57 \mathrm{kG}$

ion mass: $M=20$ electron masses

Figure $I$ shows the average electron energy as a function of time. The microwave pulse is turned on at $O$ and off at 3000 radians. The heating rate lags the power pulse because of the finite $Q$ of the cavity, which is assumed to be dominated by plasma absorption. In this case the perturbed $Q$ is about 500 . 
Figure 2 shows the amplitude of the electric potential that develops along the magnetic field. The potential initially goes negative because the preferential perpendicular electron heating causes the electrons to move toward the weak field region at the midplane. As the potential grows, the electrons execute plasma oscillations resulting in an oscillation of the potential at the electron plasma frequency. The potential is usually rather small compared to $\mathrm{kT} / \mathrm{e}$.

Figure 3 shows the average ion energy as a function of time. Note that an oscillation at the electron plasma frequency is superimposed on a monotonic growth, and that the ion energy continues to increase after the microwave pulse is tumed off (at $\left.\omega_{0} t=3000\right)$. The dashed curve is the result of a caiculation of the scochastic heating expected from the turbulent electric field in Fig. 2. This calculation was made by analysing the frequency spectrum of the space charge electric field.

Figire 4 shows the autocorrelation function of the potential in Fig. 2:

$$
A(T)=\frac{T}{T} \int_{0}^{T} \Phi(t) \Phi(t+T) d t \quad
$$

The plasma oscillation is fairly coherent, but components at other frequencies are also present:

Figure 5 shows the Fourier transform of the autocorrelation function

$$
\frac{d \Phi^{2}(\omega)}{d \omega}=\int_{-T}^{T} A(\tau) e^{i \omega T} d \tau,
$$

which is proportional to the frequency spectrum of the electric field fluctuation. Most of the power is at frequencies near the plasma frequency, but there are low-frequency components in the electric field as we11. The zero frequency component (or, more generally, the component at the ion bounce frequency) gives rise to a velocity space diffusion which leads to heating ${ }^{5}$ :

$$
\frac{d\left(k T_{i}\right)}{d t}=\frac{\pi^{2} e^{2}}{2 M} \frac{d \Phi^{2}(\omega=0)}{I^{2} d \omega} \oint \frac{n(l)}{B(l)} \sin \pi \ell / L d \ell / \oint \frac{n(l)}{B(l)} d l
$$

This stochastic heating was calculated as a function of time using the observed electric fields and the unperturbed spatial ion distribution, and the result is the dashed curve that was shown in Fig. 3.

Figure 6 shows the electron density (solid curve) and energy density (dashed curve) as a function of position along the magnetic field at the end of the run. The minimum $B$ is at $O$ and the maximum $B$ is at $I$. The resonance zone is at 0.5 . Note that the electrons, especially the energetic electrons, are confined inside the resonance surface as expected for simple, nonrelativistic, electron cyclotron heating. ${ }^{6}$

Figure 7 shows the sama curves for the ions. The ions have collapsed toward the midplane to cancel the space charge field, and the energy density is largest at an intermediate position where the space charge field is large. 
Finally, Fig. 8 shows the normalized electron and ion energy distribution at the end of the run. The solid curve is a Maxiellian. A cold electron component is observed, apparently due to electrons that always turn before reaching the resonance zone. The ion energy distribution is relatively flat up to a cutoff at about twice the average energy. The cutoff is probably due to the shortness of the computer run and to the neglect of the higher order spatial Fourier tems. The ions move a distance during the run about equal to the length of the mirror.

Some runs were also made with initially monoenergetic electrons and also with nonadiabatic electrons. The electrons were made nonadiabatic by changing the pitch angle to a new, randomly chosen value at each crossing of the mirror midplane. The usual checks of the code were made such as changing the step size, changing the number of particles, and testing for conservation of energy in the absence of an applied electric field. Different sets of random numbers were also tried with no significant change in the results.

The scaling laws for the ion heating rate have not been determined precisely, but qualitatively the heating is maximum for large microwave power, low density, short mirror length, and resonance zones near the mirror midplane.

\section{Conclusions}

In conclusion, it appears that electron cyclotron heating generally gives rise to turbulent electric fields with a component parallel to the magnetic field. Short, high-power pulses produce the most turbulence since they disturb the electron distribution more rapidly than the ions can respond. The resulting ion heating agrees very well with the velocity space diffusion calculated from the observed electric field spectrum. Other mechanisms not considered in the simulation such as ion cyclotron heating resulting from turbulent electric fields perpendicular to $\overrightarrow{\mathrm{B}}$ may also be importani. It's not obvious shat the heating observed in the simulation is the same as that observed in the toroidal octupole experiments, or that the scaling is such as to make the mechanism of interest in fusion reactors, but the effect is sufficiently enticing to warrant continued study.

\section{REFERENCES}

*Research sponsored by the U.S. Atomic Energy Commission under contract with the Union Carbide Corporation.

${ }^{2}$ G. W. Kuswa, Bull. Am. Phys. Soc. 14, 1033 (1969).

2R. Breun and J. E. Scharer, Bull. Am. Phys. Soc. 16, 1248 (1971).

3A. F. Kuckes, Plasma Phys. 10, 367 (1968).

4. C. Sprott and P. E. Edmonds 14, 2703 (1971).

5. Puri, Phys. Fluids 11, 1745 (1968).

${ }^{6}$ G. E. Guest, Bull. Am. Phys. Soc. 13, 314 (1968). 
ION ENERGT (EV)

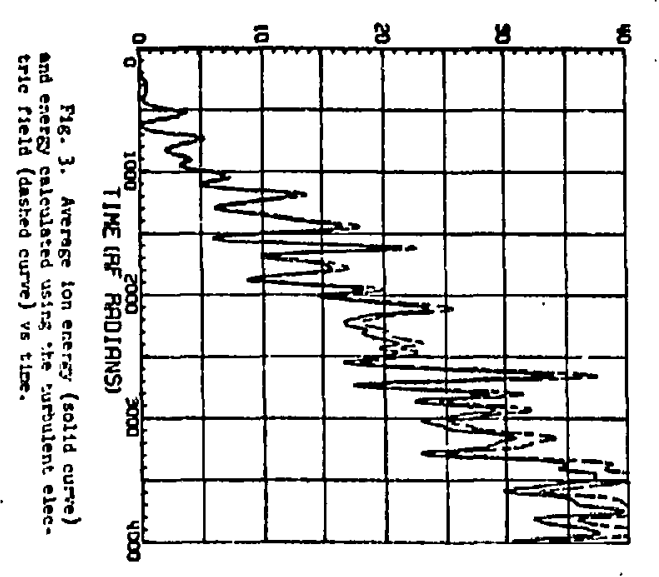

POTENTIAL CORRELATION FCN (SQ KV)

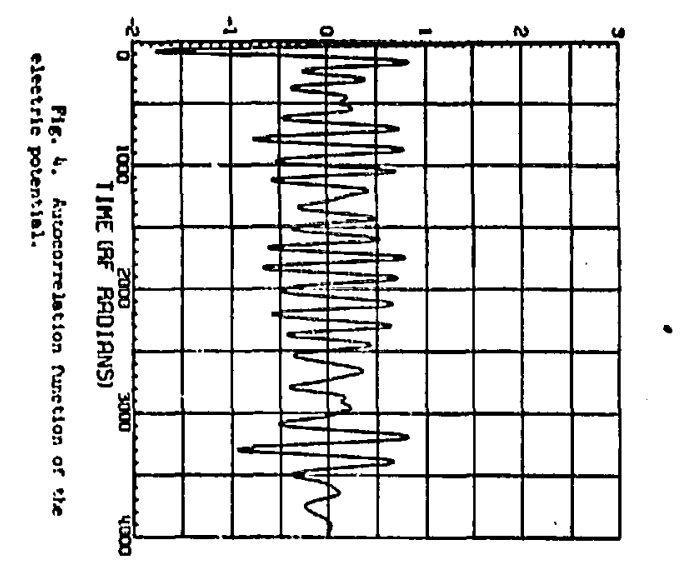

ELECTRON ENERGT IKEV

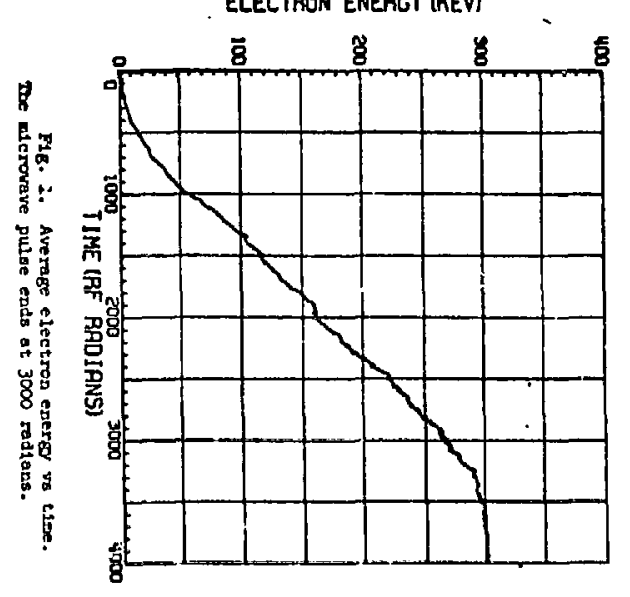

ELECTAIC POTENTIAL (KILOVOLTS)

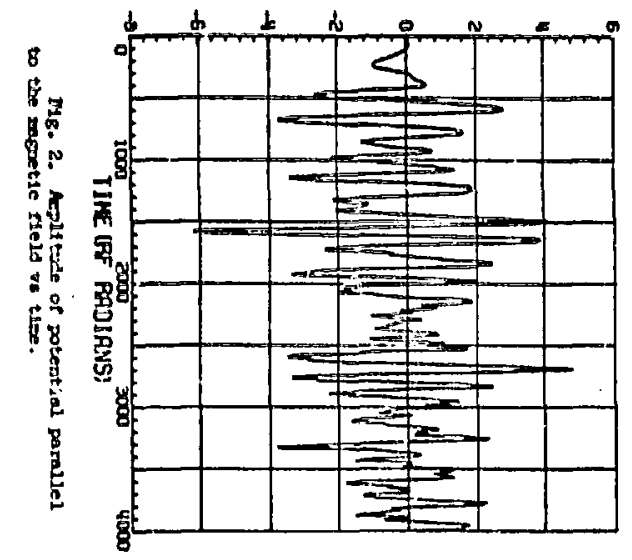




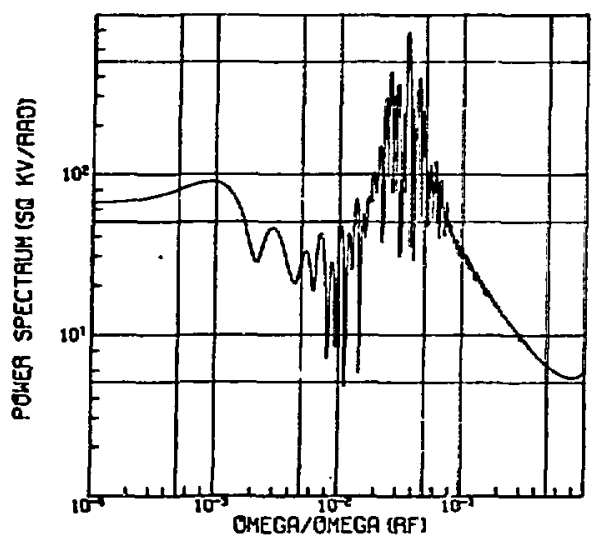

Fig. 5. Forer spectrom of the turbulest elece trie sield vo srequency. The electron pioes srequeney is at $w=3 \times 10^{-2} \omega_{0}$.

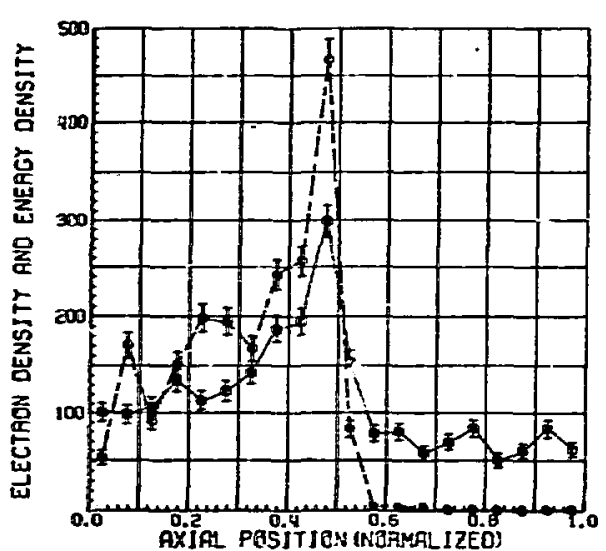

P1c. 5. Eitectres leseity (solic curre) and eneror censity (axsyed eurse) os position alore tre Field at atre end of the nin. The field tini Ene is at $C$ aftd the bexies 15 at 1.0 . he reomance zoxe 1 is at 0.5 .

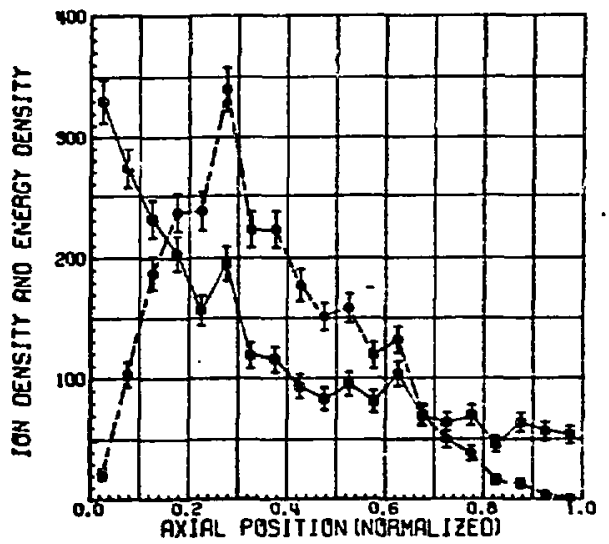

P1g. 7. Ion density (solid eurve) and enere denulty (dashed curre) vo position alors the tield at the of ere tor. and the paximn 1s at 1.0. The resoname zore is atd 0.5 .

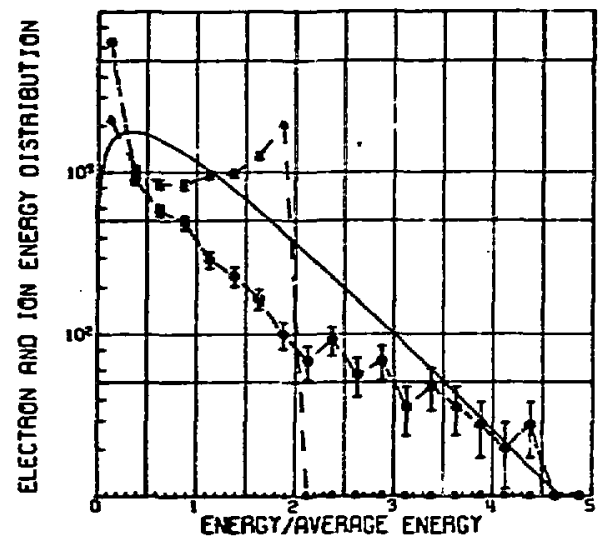

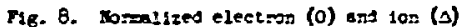

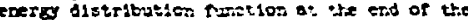
ne. The solsd curre is a Murellier. 